\title{
2p2h effects on the weak pion production cross section
}

\author{
A. Mariano and C. Barbero \\ Departamento de Física, Facultad de Ciencias Exactas, \\ Universidad Nacional de La Plata, Argentina. \\ Instituto de Física La Plata, CONICET, Argentina.
}

\begin{abstract}
The $v_{l} n \rightarrow l^{-} p \mathrm{QE}$ reaction on the A-target is used as a signal event or/and to reconstruct the neutrino energy, using two-body kinematics. Competition of another processes could lead to misidentification of the arriving neutrinos, being important the fake events coming from the $\mathrm{CC} 1 \pi$ background. A precise knowledge of cross sections is a prerequisite in order to make simulations in event generators to substract the fake ones from the QE countings, and in this contribution we analyze the different nuclear effects on the $\mathrm{CC} 1 \pi$ channel. Our calculations also can be extended for the NC case.
\end{abstract}

Keywords: neutrino, background, pion production

PACS: $13.15 .+\mathrm{g}, 13.60 . \mathrm{Le}$

$v_{\mu} \rightarrow v_{x}$ disappearance experiments uses $v_{\mu} n \rightarrow \mu^{-} p$ CCQE to detect neutrinos and reconstruct its energy. $E_{v}$ determination could be wrong for a fraction of $\mathrm{CC} 1 \pi^{+}$background events $(20 \%) v_{\mu} p \rightarrow \mu^{-} p \pi^{+}$, that can mimic a CCQE one if the pion is absorbed and/or not detected. These processes take place into the target nucleus and nuclear effects as smearing $(\mathrm{S})$ of the reconstructed energy by the momentum distribution $\left(n_{A}\right)$ of the target binding (B) nucleons, should be taken into account. In addition final state interactions (FSI) of the emerging hadrons generate energy lost,change of direction,charge transfer or multiple nucleon knock out(np-nh). Finally meson exchange currents (MEC) processes lead to additional contributions to one-body current generated. In what follows we concentrate on the $2 \mathrm{p} 2 \mathrm{~h}+1 \pi$ contributions to the pion production cross section, and compare with the $1 \mathrm{p} 1 \mathrm{~h}+1 \pi$ one already analyzed previously [1]. The $2 \mathrm{p} 2 \mathrm{~h}+1 \pi$ amplitude is depicted in Figure 1 and the corresponding differential cross section reads $\left(\mathscr{N}^{2}(k) \equiv \frac{f}{(2 \pi)^{3} E(k)}, f=1 / 2(M)\right.$ for bosons(fermions))

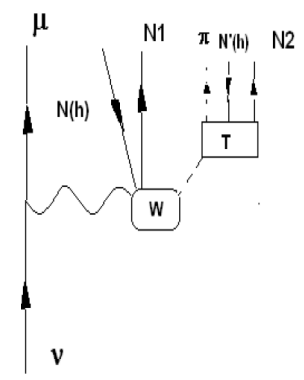

FIGURE 1. Amplitude for the $v N \rightarrow l N^{\prime} N_{1} N_{2} \pi(2 p 2 h+1 \pi)$ process.

$$
\begin{aligned}
& \quad d \sigma_{v A}^{2 p 2 h+1 \pi}=\frac{n_{A}(N)\left(1-n_{A}\left(N_{1}\right)\right)}{\left|\mathbf{v}_{v}-\mathbf{v}_{A}\right| 2 E(v)} \frac{1}{2} \sum_{m_{v}, m_{N}, m_{l}, m_{N_{1}}} \int \frac{d^{4} \pi^{\prime}}{(2 \pi)^{4}}\left[\mathscr{S} \sum_{m_{N_{2}} m_{N}^{\prime}}\left|T\left(N_{2} m_{N_{2}}, \pi, N^{\prime} m_{N^{\prime}}, \pi^{\prime}\right)\right|^{2} \frac{n_{A}\left(N^{\prime}\right)\left(1-n_{A}\left(N_{2}\right)\right)}{\left(\pi^{\prime 2}-M_{\pi}^{2}\right)^{2}}\right. \\
& \left.\times \quad(2 \pi)^{4} \delta^{4}\left(N_{2}+\pi-\pi^{\prime}-N^{\prime}\right) d^{3} N_{2} \mathscr{N}^{2}\left(N_{2}\right) d^{3} N^{\prime} \mathscr{N}\left(N^{\prime}\right) d^{3} \pi \mathscr{N}^{2}(\pi)\right]\left|W^{\mu}\left(N_{1} m_{N_{1}}, l m_{l}, \pi^{\prime}, N m_{N}, v m_{v}\right) J_{\mu}^{l}\left(l m_{l}, v m_{v}\right)\right|^{2} \\
& \times \quad(2 \pi)^{4} \delta^{4}\left(N_{1}+\pi^{\prime}+l-v-N\right) d^{3} l \mathscr{N}^{2}(l) d^{3} N_{1} \mathscr{N}^{2}\left(N_{1}\right) d^{3} N \mathscr{N}(N),
\end{aligned}
$$

being $N, \pi, l, v \equiv(E(N, \pi, l, v), N, \pi, l, v), E(k)=\sqrt{k^{2}+M^{2}}, m \equiv \operatorname{spin}, \mathscr{S}$ symmetrization factor, $\mathrm{T} \pi^{\prime} N^{\prime} \rightarrow \pi N_{2}$ rescattering is simplified replacing

$$
[\cdots] \Rightarrow \frac{1}{\left(\pi^{\prime 2}-M_{\pi}^{2}-\Pi\left(\pi^{\prime}\right)\right)} \Rightarrow 2 \pi \delta\left(\pi^{\prime 2}-M_{\pi}^{2}-\mathfrak{R}\left(\Pi\left(\pi^{\prime}\right)\right)\right)
$$


as shown in Figure 2.

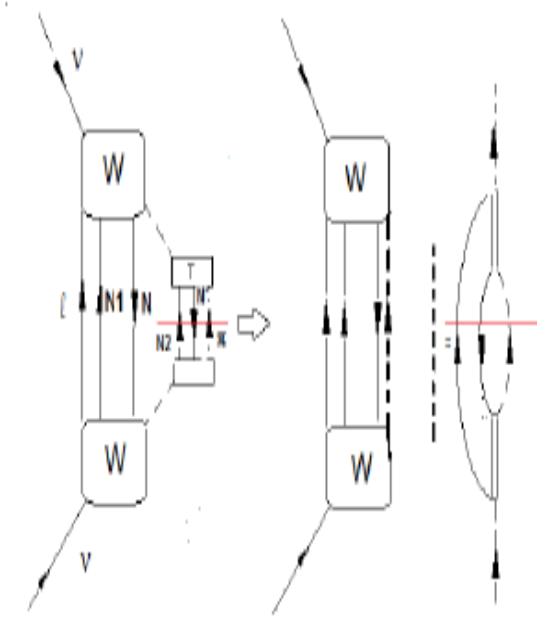

FIGURE 2. Simplification to calculate $\pi^{\prime} N^{\prime}(h)$ rescattering.

Figure 3 shows results for the total $\mathrm{CC} 1 \pi^{+}$cross section and the gradual effect of the B, S and FSI within the $1 \mathrm{p} 1 \mathrm{~h}+1 \pi$ configuration space. Also the results for $B+S+F S I$ in the $2 p 2 h+1 \pi$ one and full $1 p 1 h+2 p 2 h+1 \pi$ one are shown. They are also shown for the differential cross section in Figure 4.B effects are considered within the Relativistic Hartree approximation (RHA) of QHD I [2], for $\mathrm{N}$ and $\Delta$ using universal couplings. $n_{A}$ is obtained from a perturbative approach in nuclear matter within a $2 \mathrm{p} 2 \mathrm{~h}+4 \mathrm{p} 4 \mathrm{~h}$ configuration space[3]. FSI on nucleons is taken (Toy model !) through the RHA effective fields also for final N, while for pions we use the Eikonal approach[4].
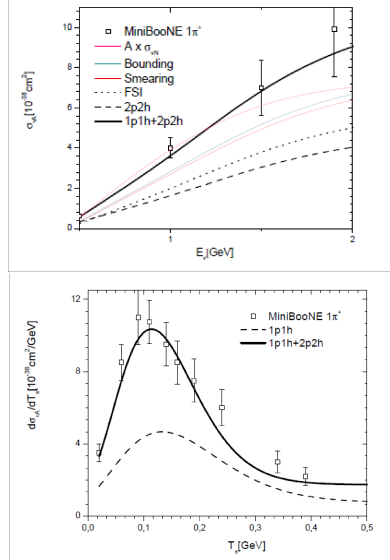

FIGURE 3. Up: Total $1 \pi^{+}$cross section compared with MiniBooNE data (see[1]). Down: $1 \pi^{+}$production differential cross section.

We conclude that $2 \mathrm{p} 2 \mathrm{~h}$ contribution is important and comparable to the $1 \mathrm{p} 1 \mathrm{~h}$ one. In the $\pi^{0}$ channel results are not so good in reproducing the data and nonresonant contributions and charge exchange terms should be included in the rescattering amplitude. Finally, MEC should be included at the same time that $2 \mathrm{p} 2 \mathrm{~h}$ contributions in order to have a more real estimation.

\section{ACKNOWLEDGMENTS}

The assistance of A.M. to the NuFACT 2012 was supported by the UNLP under the fellowship for trips 2012. 


\section{REFERENCES}

1. O. Lalakulich and U. Mosel, arXiv:1210.4717 [nucl-th] (2012).

2. B.D. Serot and J.D. Walecka, Adv. Nucl. Phys.16,(1986) 1.

3. A. Mariano, F. Krmpotić and A.F.R de Toledo Piza, Phys. Rev. C53,(1996)1.

4. C. Barbero, A. Mariano, and S. B. Duarte, Phys. Rev. C, 82 (2010) 067305. 